\title{
Survey on Physicians' Knowledge and Training Needs in Genetic Counseling in Germany
}

\author{
Julia Dick ${ }^{a}$ Viktoria Aue $^{a}$ Simone Wesselmann ${ }^{b}$ Anne Brédartt ${ }^{c, d}$ \\ Sylvie Dolbeaultc, e Peter Devilee ${ }^{f}$ Dominique Stoppa-Lyonnet ${ }^{g}$ \\ Rita K. Schmutzler ${ }^{a}$ Kerstin Rhiem ${ }^{a}$ \\ ${ }^{a}$ Center for Hereditary Breast and Ovarian Cancer and Center for Integrated Oncology (CIO), Medical Faculty, \\ University Hospital Cologne, Cologne, Germany; ${ }^{b}$ German Cancer Society (DKG), Berlin, Germany; \\ 'Supportive Care Department, Psycho-Oncology Unit, Institut Curie, Paris, France; dUniversity Paris Descartes, \\ Boulogne-Billancourt, France; ${ }^{e}$ Centre de Recherche en Épidémiologie et Santé des Populations (CESP), \\ University Paris-Sud, UVSQ, INSERM, University Paris-Saclay, Villejuif Cedex, France; ${ }^{\mathrm{f} D e p a r t m e n t s ~ o f ~ H u m a n ~}$ \\ Genetics and Pathology, Leiden University Medical Centre, Leiden, The Netherlands; ${ }^{9}$ Cancer Genetic Clinic, \\ Institut Curie, Paris, France
}

\section{Keywords \\ $B R C A 1 / B R C A 2$ mutations · Hereditary breast cancer . \\ Genetic counseling · Genetic testing}

\section{Abstract}

Background: In recent years, germline testing of women with a risk of developing breast and ovarian cancer has increased rapidly. This is due to lower costs for new highthroughput sequencing technologies and the manifold preventive and therapeutic options for germline mutation carriers. The growing demand for genetic counseling meets a shortfall of counselors and illustrates the need to involve the treating clinicians in the genetic testing process. This survey was undertaken to assess their state of knowledge and training needs in the field of genetic counseling and testing. Methods: A cross-sectional survey within the European Bridges Study (Breast Cancer Risk after Diagnostic Gene Sequencing) was conducted among physician members ( $n=$ 111) of the German Cancer Society who were primarily gynecologists. It was designed to examine their experience in genetic counseling and testing. Results: Overall, the study revealed a need for training in risk communication and clinical recommendations for persons at risk. One-third of re-
\end{abstract}

spondents communicated only relative disease risks (31.5\%) instead of absolute disease risks in manageable time spans. Moreover, almost one-third of the respondents (31.2\%) communicated bilateral and contralateral risk-reducing mastectomy as an option for healthy women and unilateral-diseased breast cancer patients without mutations in high-risk genes (e.g. BRCA1 or BRCA2). Most respondents expressed training needs in the field of risk assessment models, the clinical interpretation of genetic test results, and the decisionmaking process. Conclusion: The survey demonstrates a gap of genetic and risk literacy in a relevant proportion of physicians and the need for appropriate training concepts.

๑) 2020 S. Karger AG, Basel

\section{Introduction}

Up to $30 \%$ of breast cancer patients fulfill the familial criteria for genetic testing of the German Consortium for Hereditary Breast and Ovarian Cancer (GC-HBOC) [1]. Within the GC-HBOC [2], a comprehensive care concept for families at risk has been in place since 1996. This ranges from individual risk calculation, standardized counseling, and genetic testing, to a nondirective decision-mak- 
ing process on risk-adjusted preventive measures (e.g., intensified surveillance and risk-reducing surgery).

Of the patients who fulfil the criteria of the GC-HBOC, $24 \%$ carry deleterious germline mutations in $B R C A 1$ or $B R C A 2$ [3] and face a considerably elevated lifetime risk of developing breast and ovarian cancer $[4,5]$. As they can choose from a wide range of specific preventive and therapeutic options (e.g., PARPi) [2,6-14] and, at the same time the costs of genetic testing are steadily decreasing, a significantly higher demand for genetic counseling can be expected or is already observed [15]. However, recent studies indicate that a considerable number of patients are denied testing or have no access to it $[16,17]$. This could possibly be due to the insufficient genetic literacy of physicians as well as the lack of genetic counsellors [17, 18].

We conducted a cross-sectional survey of members of the German Cancer Society (GCS) to evaluate physicians' understanding of genetic cancer risk and preventive options as a prerequisite for appropriate counseling. Within the European BRIDGES (Breast Cancer Risk after Diagnostic Gene Sequencing) research program, aimed at developing and implementing a comprehensive breast cancer risk assessment model, Brédart et al. $[19,20]$ performed an initial survey focusing on genetic professionals registered to use the online risk assessment model BOADICEA (Breast and Ovarian Analysis of Disease Incidence and Carrier Estimation Algorithm) [21]. Specifically, counselors' perceived importance of breast cancer risk factors as well as the usability and acceptance of BOADICEA were examined.

In this study, we present a complementary survey aimed primarily at health-care professionals carrying out genetic counseling, and provide insights into their knowledge of cancer risks, genetics, and training needs. Based on these survey results, existing qualification programs for genetic practice can be extended and adapted to physicians' needs.

\section{Materials and Methods}

This cross-sectional survey was performed on members of the German Cancer Society as part of the European BRIDGES research program (https://bridges-research.eu) between October 2016 and January 2017. They were invited to participate in an online survey via e-mail. The survey link was accessed 185 times, and a total of 111 questionnaires were partially or completely filled out.

\section{Questionnaire}

The online questionnaire (open-source LimeSurvey) was developed at the Center for Hereditary Breast and Ovarian Cancer at the University Hospital in Cologne on 6 topics. (1) Assessment of physicians' experience in genetic counseling and testing of people at risk for hereditary breast and ovarian cancer. (2) Physicians' assessment of the importance of breast cancer risk factors, and the utilization of cancer genetic risk guidelines and prediction models (e.g., the Claus model, Cyrillic, and BOADICEA). (3) Physicians' advice on clinical risk management for (a) women carrying pathogenic variants in the BRCA1/2 genes, (b) women without pathogenic variants but a remaining lifetime risk of developing breast cancer of $>30 \%$, and (c) women without pathogenic variants but a remaining lifetime risk of developing breast cancer of $15-30 \%$. Furthermore, physicians were asked to indicate which common diagnostic methods they use as part of the intensified breast cancer surveillance program, and whether they usually provide psychological consultation to support decision-making about preventive options. (4) The usefulness and usability of the BOADICEA Web-based risk assessment model. (5) Questions about the information and training needs of physicians in the familial breast and ovarian cancer setting. (6) Demographic data such as age, gender, and profession as well as information on participants' clinical practice.

\section{Statistical Analysis}

Data analysis was based on 111 questionnaires. Not all questions were answered by all participants, resulting in a different number of answers per question. Analysis was performed with the Statistical Package for Social Sciences (SPSS v25.0). Descriptive data were extracted from frequency tables. the $\chi^{2}$ test was applied for categorical variables to determine the difference between 2 groups. Statistical significance was set at $p=0.05$. All $p$ values reported were two-tailed.

\section{Results}

Physicians' Experience in Genetic Counseling and Risk Communication

Of the 111 respondents, $81.1 \%$ ( $n=90)$ used the checklist of the GC-HBOC to assess the family burden [1]. Physicians' participation in specific training was significantly related to the consideration of family-based inclusion criteria $\left(\chi^{2}[2]=17.014 ; p<0.001\right)$ but was not significantly associated with offering psychological consultation for women at high risk $\left(\chi^{2}[1]=2.021 ; p=0.155\right)$. A total of $92(82.8 \%)$ completed the questions about communicating breast and ovarian cancer risks; $31.5 \%$ of these communicated relative risks, $43.5 \%$ absolute life-time risks, and $30.4 \%$ absolute risks in manageable time spans (e.g., in the next 5,10 , or 15 years).

Personal and qualification details were voluntary and were provided by 74 of the 111 (66.6\%) participants in the survey. Most participants were gynecologists (69/74), $54.1 \%(n=40)$ of whom indicated that they had received specific training in genetic testing and counseling for hereditary cancer diseases in GC-HBOC centers, but $45.9 \%(n=34)$ did not receive any training. A majority of $68.9 \%$ of participants $(51 / 74)$ specified that they spent $>80 \%$ of their monthly working time providing patient care. Of these, $58.8 \%(n=30)$ physicians provided risk counseling to $<5$ patients a week. Seventy-one of the 74 participants provided information on gender and age; there were 36 men and 35 women and 40 were at least 50 years old. 


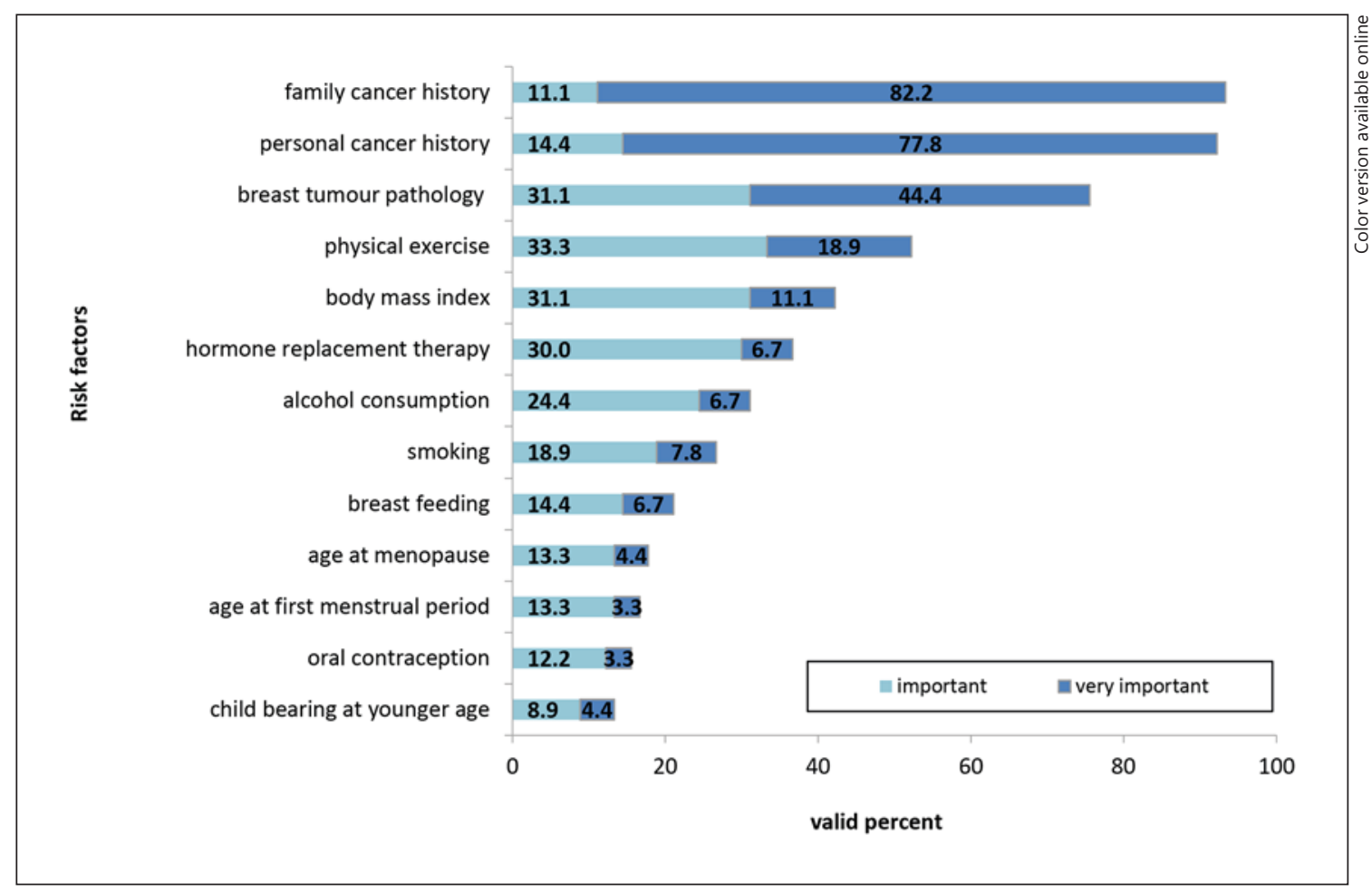

Fig. 1. Percentages of physicians considering risk factors to be important or very important on a 5-point Likert Scale (unimportant, less important, neither unimportant nor important, important, very important) for estimating breast cancer risk $(n=90)$.

Importance of Factors for Estimating Breast Cancer

Risk and Use of Cancer Risk Prediction Models

Questions on the importance of breast cancer risk factors were answered by 90 of the 111 respondents. Most of the physicians primarily took patients' family (93.3\%) and personal cancer histories (92.2\%) into consideration for assessing breast cancer risk. Breast tumor pathology was rated to be either very important or important by $75.5 \%$. Further cancer risk factors are represented in Figure 1 below.

A total of 88 (79.2\%) of the 111 participants provided answers about the use of cancer risk prediction models. Most of the risk prediction models were unknown to the physicians (Fig. 2); the Myriad-BRCAtool was not known by $44.3 \%(n=39)$ and the Eisinger score was not known by $60.2 \%(n=53)$. Even when the risk prediction models were known, in most cases they were never used. BOADICEA was not used by $29.2 \%$ of physicians who knew the model. Only a small proportion of physicians used a model occasionally or regularly to estimate breast cancer risk.

\section{Advice on Clinical Risk Management}

Information about risk-reducing options was provided by 77 of 111 respondents. Tables 1 and 2 present the preventive options that physicians considered for counseling women carrying pathogenic variants in $B R C A 1$ and BRCA2 as well as women without pathogenic variants but with a lifetime risk of $\geq 30 \%$ of developing breast and ovarian cancer. Transvaginal ultrasound and determination of the tumor marker CA125 were both considered to be efficient preventive options for the early detection of ovarian cancer by 59.7 and $19.5 \%$ of physicians, respectively. Similarly, $77.9 \%$ of physicians discussed "secondary prophylactic mastectomy" of the diseased breast as a risk-reducing option in BRCA1/2 mutation carriers with unilateral breast cancer. Prophylactic mastectomies and salpingo-oophorectomies were discussed by one-third of physicians as risk-reducing options for breast cancer in women with an increased risk but without pathogenic variants of the risk genes.

\section{Training Needs}

Information about training needs was provided by 74 of the 111 participants. Many physicians indicated a need for training in counseling on preventive options after variants of uncertain significance (VUS) $(50.0 \%)$ were identified, the application of breast cancer risk calculation models (43.2\%), and the clinical interpretation of test results (39.2\%). Further training needs are outlined in Figure 3. 


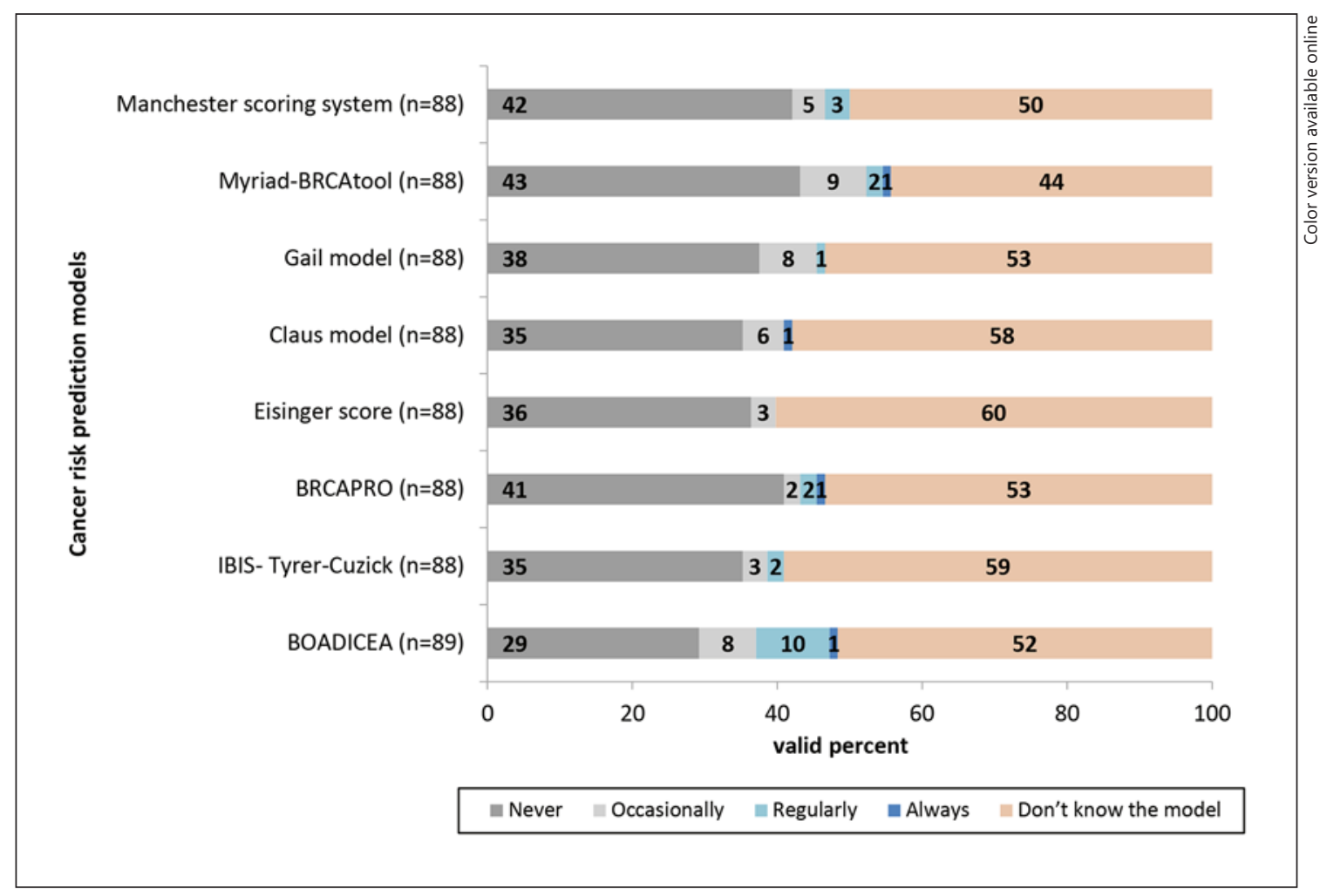

Fig. 2. Percentages of physicians using cancer risk prediction models.

Table 1. Risk-reducing options for breast cancer considered by physicians for carriers of $B R C A 1 / 2$ pathogenic variants and women at an increased risk but without pathogenic variants $(n=77)$

\begin{tabular}{|c|c|c|c|}
\hline Preventive options & $\begin{array}{l}B R C A 1 / 2 \\
\text { carriers }\end{array}$ & $\begin{array}{l}\text { Women with a } \\
\text { lifetime risk of } \geq 30 \%\end{array}$ & $\begin{array}{l}\text { Women with a } \\
\text { lifetime risk of } 15-30 \%\end{array}$ \\
\hline Prophylactic bilateral mastectomy in healthy women & $94.8 \%$ & $31.2 \%$ & $18.2 \%$ \\
\hline $\begin{array}{l}\text { Prophylactic contralateral mastectomy in women } \\
\text { with unilateral breast cancer }\end{array}$ & $77.9 \%$ & $31.2 \%$ & $19.5 \%$ \\
\hline $\begin{array}{l}\text { Prophylactic bilateral mastectomy in women with } \\
\text { unilateral breast cancer }\end{array}$ & $77.9 \%$ & $32.5 \%$ & $16.9 \%$ \\
\hline Prophylactic bilateral salpingo-oophorectomy & $93.5 \%$ & $26.0 \%$ & $18.2 \%$ \\
\hline Intensified breast cancer surveillance & $94.8 \%$ & $98.7 \%$ & $89.6 \%$ \\
\hline Mammography & $95.9 \%$ & $96.1 \%$ & $95.7 \%$ \\
\hline Sonography & $98.6 \%$ & $98.7 \%$ & $98.6 \%$ \\
\hline MRI & $90.4 \%$ & $78.9 \%$ & $72.5 \%$ \\
\hline Chemoprevention (e.g., tamoxifen) & $31.2 \%$ & $19.5 \%$ & $156 \%$ \\
\hline Lifestyle interventions & $71.4 \%$ & $66.2 \%$ & $71.4 \%$ \\
\hline
\end{tabular}

Table 2. Risk-reducing options for ovarian cancer considered by physicians for carriers of $B R C A 1 / 2$ pathogenic variants and women at an increased risk but without pathogenic variants $(n=77)$

\begin{tabular}{llll}
\hline Clinical options & $\begin{array}{l}\text { Carriers of } \\
\text { BRCA1/2 }\end{array}$ & $\begin{array}{l}\text { Women with a } \\
\text { lifetime risk of } \geq 30 \%\end{array}$ & $\begin{array}{l}\text { Women with a } \\
\text { lifetime risk of 15-30\% }\end{array}$ \\
\hline $\begin{array}{l}\text { Prophylactic bilateral salpingo-oophorectomy } \\
\text { Ultrasound examination of the ovaries }\end{array}$ & $\begin{array}{l}97.4 \% \\
59.7 \%\end{array}$ & $\begin{array}{l}70.1 \% \\
63.6 \%\end{array}$ \\
CA125 marker measurement & $19.5 \%$ & $64.9 \%$ \\
\hline
\end{tabular}




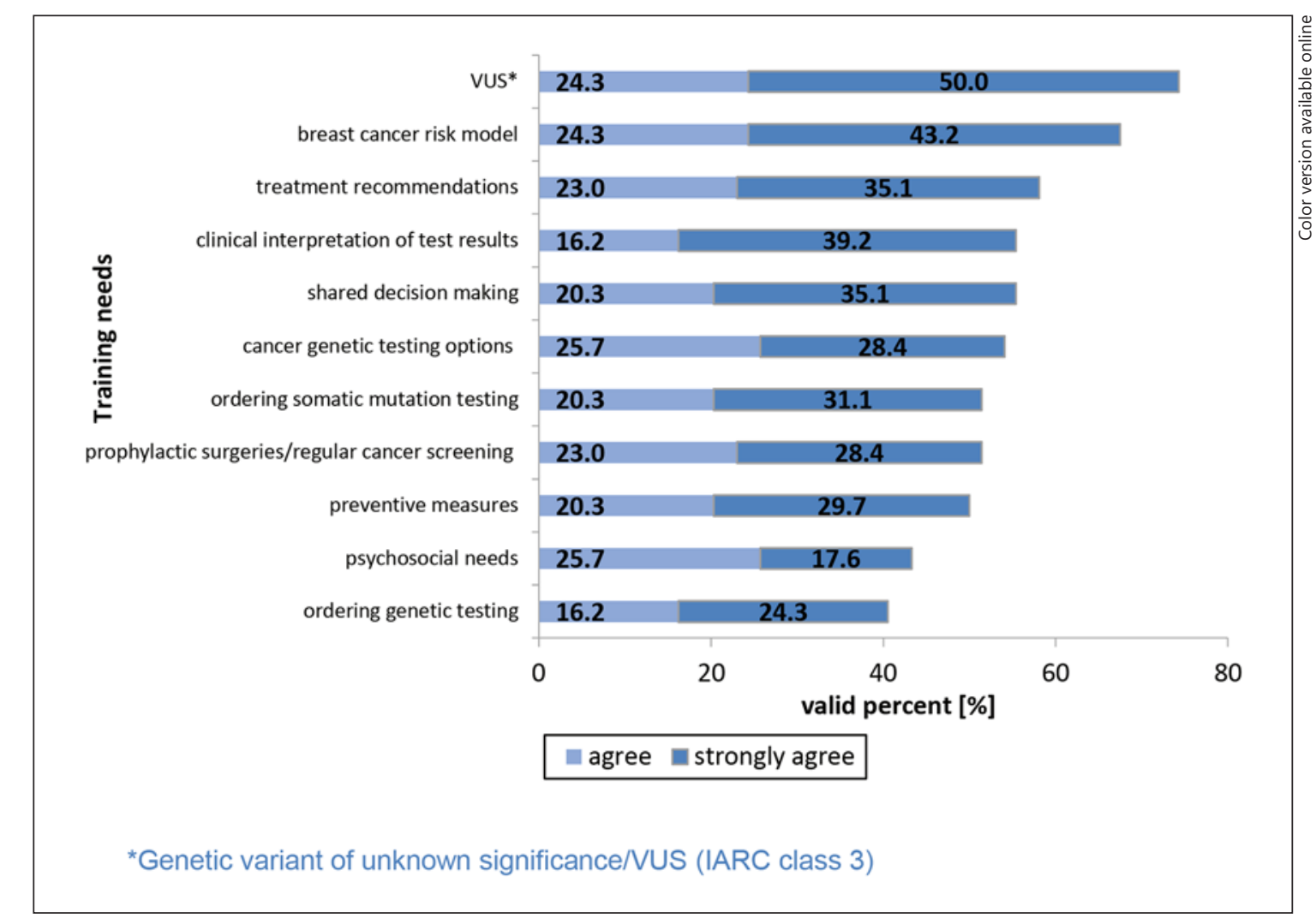

Fig. 3. Training needs that participants either agreed or strongly agreed with $(n=74)$.

\section{Discussion}

Our survey highlights important findings on physicians' knowledge about genetics, cancer risk communication, and the clinical management of at-risk patients, i.e., knowledge that can improve their competence in risk counseling. The majority of respondents were aware of the rapidly increasing value of genetic testing in oncology with regard to therapeutic and preventive options, and screened their patients for hereditary cancer burden by using the national inclusion criteria and the checklist developed for this purpose $[1,22]$. However, recent studies show that only a minority of patients who comply with the criteria actually undergo genetic testing $[16,23]$. This is partly due the persisting shortfall of genetic counsellors and the limited genetic literacy of clinicians [24, 25]. Closing this gap in genetic literacy is getting more critical, now that the demand for counseling is increasing, e.g., due to the relevance for treatment (e.g., of PARP inhibitors) $[12-14]$, the uptake of somatic tumor profiling, germline testing, and technological advances in genetic testing.

The improvement of genetic literacy is an interdisciplinary challenge for the entire oncology team. For example, a study showed a rate of genetic testing by surgeons that varied between 26.3 and $72.3 \%$, depending, among other factors, on their own attitude towards testing [26]. Many surgeons stated that they rarely or never allow the promise of genetic test results to delay surgery, despite the fact that, for instance, risk-reducing contralateral mastectomy improves the survival of $B R C A 1 / 2$ carriers and could be integrated into the surgical management [27]. Different approaches such as education programs, molecular tumor boards, and enhanced clinical decision support tools may improve genetic literacy within an oncology team [28].

One-third of the respondents communicated bilateral and contralateral prophylactic mastectomy as a risk-reducing surgical option for women without pathogenic variants in $B R C A 1 / 2$. While women with deleterious germline variants in $B R C A 1 / 2$ may benefit from risk-reducing mastectomy [7], this has not yet been shown in women without mutations in high-risk genes. This option is thus usually not available to these patients. In a population-based study in California, USA, it was found that bilateral mastectomy was increasingly used in women newly diagnosed with unilateral breast cancer, but that there was no survival benefit associated with bilateral mastectomy when compared to unilateral breast-conserving surgery [29]. The number of mastectomies within the group of women without mutations nevertheless increased for several reasons. 
While $97 \%$ of the respondents indicated that prophylactic salpingo-oophorectomy reduces the ovarian cancer risk, nearly $60 \%$ of the respondents stated that they consider vaginal ultrasound in combination with CA125 tumor marker determination (19.5\%) when counseling $B R C A 1$ and BRCA2 mutation carriers. While the importance of a risk-reducing removal of the ovaries and fallopian tubes is beyond doubt [30], screening measures for ovarian carcinoma are proven inefficient [31].

The majority of respondents expressed a need for further training in the application of risk assessment models, the clinical interpretation of genetic test results (especially of variants of unknown significance) and communicating about risks. This also reflects the fact that the results of genetic testing are becoming increasingly complex as the prevalence of variants of uncertain significance in panel testing is significantly increasing, and the clinical implications become more diverse when pathogenic variants are found in the growing list of genes being tested [32].

\section{Conclusion}

Our findings demonstrate a deficit in genetic counseling expertise among physicians. Although there may be country-specific differences and the level of genetic literacy has increased in the meantime, recent studies support the remaining training need identified in our study. Education programs are needed for risk assessment, the understanding and communication of genetic test results, and risk-adjusted clinical recommendations [33]. The de- velopment and implementation of programs to educate the treating physicians about predictive and diagnostic genetics will improve the care of patients and outcomes.

\section{Acknowledgements}

We would like to thank all participants from the German Cancer Society who took part in the survey.

\section{Statement of Ethics}

Ethics vote is not required.

\section{Conflict of Interest Statement}

The authors declare there are no conflicts of interest.

\section{Funding Sources}

As part of the BRIDGES project, this work was funded by the European Union Horizon 2020 research and innovation program with the grant number No. 634935. It was also co-financed by the Center for Hereditary Breast and Ovarian Cancer at the University Hospital in Cologne, where the survey was conducted.

\section{Author Contributions}

R.K.S. and K.R. developed the concept and questionnaire. All authors analysed and interpreted the data, and prepared and reviewed the manuscript.

\section{References}

1 Rhiem K, Bücker-Nott HJ, Hellmich M, Fischer H, Ataseven B, Dittmer-Grabowski C, et al. Benchmarking of a checklist for the identification of familial risk for breast and ovarian cancers in a prospective cohort. Breast J. 2019 May;25(3):455-60.

2 Bick U, Engel C, Krug B, Heindel W, Fallenberg EM, Rhiem K, et al.; German Consortium for Hereditary Breast and Ovarian Cancer (GC-HBOC). High-risk breast cancer surveillance with MRI: 10-year experience from the German consortium for hereditary breast and ovarian cancer. Breast Cancer Res Treat. 2019 May;175(1):217-28.

3 Kast K, Rhiem K, Wappenschmidt B, Hahnen E, Hauke J, Bluemcke B, et al.; German Consortium for Hereditary Breast and Ovarian Cancer (GC-HBOC). Prevalence of BRCA1/2 germline mutations in 21401 families with breast and ovarian cancer. J Med Genet. 2016 Jul;53(7):465-71.

4 Kuchenbaecker KB, Hopper JL, Barnes DR, Phillips KA, Mooij TM, Roos-Blom MJ, et al.; BRCA1 and BRCA2 Cohort Consortium.
Risks of Breast, Ovarian, and Contralateral Breast Cancer for BRCA1 and BRCA2 Mutation Carriers. JAMA. 2017 Jun;317(23):240216.

5 Engel C, Fischer C, Zachariae S, Bucksch K, Rhiem K, Giesecke J, et al.; German Consortium for Hereditary Breast and Ovarian Cancer (GC-HBOC). Breast cancer risk in BRCA1/2 mutation carriers and noncarriers under prospective intensified surveillance. Int J Cancer. 2020 Feb;146(4):999-1009.

6 Meijers-Heijboer H, van Geel B, van Putten WL, Henzen-Logmans SC, Seynaeve C, Menke-Pluymers MB, et al. Breast cancer after prophylactic bilateral mastectomy in women with a BRCA1 or BRCA2 mutation. N Engl J Med. 2001 Jul;345(3):159-64.

7 Domchek SM, Friebel TM, Singer CF, Evans DG, Lynch HT, Isaacs C, et al. Association of risk-reducing surgery in BRCA1 or BRCA2 mutation carriers with cancer risk and mortality. JAMA. 2010 Sep;304(9):967-75.

8 Li X, You R, Wang X, Liu C, Xu Z, Zhou J, et al. Effectiveness of Prophylactic Surgeries in
BRCA1 or BRCA2 Mutation Carriers: A Meta-analysis and Systematic Review. Clin Cancer Res. 2016 Aug;22(15):3971-81.

9 Kauff ND, Domchek SM, Friebel TM, Robson ME, Lee J, Garber JE, et al. Risk-reducing salpingo-oophorectomy for the prevention of BRCA1- and BRCA2-associated breast and gynecologic cancer: a multicenter, prospective study. J Clin Oncol. 2008 Mar;26(8): 1331-7.

10 Tutt A, Robson M, Garber JE, Domchek SM, Audeh MW, Weitzel JN, et al. Oral poly(ADP-ribose) polymerase inhibitor olaparib in patients with BRCA1 or BRCA2 mutations and advanced breast cancer: a proof-of-concept trial. Lancet. $2010 \mathrm{Jul}$; 376(9737):235-44.

11 Audeh MW, Carmichael J, Penson RT, Friedlander M, Powell B, Bell-McGuinn KM, et al. Oral poly(ADP-ribose) polymerase inhibitor olaparib in patients with BRCA1 or BRCA2 mutations and recurrent ovarian cancer: a proof-of-concept trial. Lancet. 2010 Jul; 376(9737):245-51. 
12 Moore K, Colombo N, Scambia G, Kim BG, Oaknin A, Friedlander M, et al. Maintenance Olaparib in Patients with Newly Diagnosed Advanced Ovarian Cancer. N Engl J Med. 2018 Dec;379(26):2495-505.

13 Robson M, Im SA, Senkus E, Xu B, Domchek SM, Masuda N, et al. Olaparib for Metastatic Breast Cancer in Patients with a Germline BRCA Mutation. N Engl J Med. 2017 Aug; 377(6):523-33

14 Litton JK, Rugo HS, Ettl J, Hurvitz SA, Gonçalves A, Lee KH, et al. Talazoparib in Patients with Advanced Breast Cancer and a Germline BRCA Mutation. N Engl J Med. 2018 Aug; 379(8):753-63.

15 Kurian AW, Ford JM. Multigene Panel Testing in Oncology Practice: How Should We Respond? JAMA Oncol. 2015 Jun;1(3):277-8.

16 Childers CP, Childers KK, Maggard-Gibbons M, Macinko J. National Estimates of Genetic Testing in Women With a History of Breast or Ovarian Cancer. J Clin Oncol. 2017 Dec; 35(34):3800-6.

17 Kurian AW, Li Y, Hamilton AS, Ward KC, Hawley ST, Morrow M, et al. Gaps in Incorporating Germline Genetic Testing Into Treatment Decision-Making for Early-Stage Breast Cancer. J Clin Oncol. 2017 Jul;35(20) 2232-9.

18 Cooksey JA, Forte G, Flanagan PA, Benkendorf J, Blitzer MG. The medical genetics workforce: an analysis of clinical geneticist subgroups. Genet Med. 2006 Oct;8(10):60314.

19 Brédart A, Kop JL, Antoniou AC, Cunningham AP, De Pauw A, Tischkowitz M, et al. Use of the BOADICEA Web Application in clinical practice: appraisals by clinicians from various countries. Fam Cancer. 2018 Jan 17(1):31-41.
20 Brédart A, Kop JL, Antoniou AC, Cunningham AP, De Pauw A, Tischkowitz M, et al. Clinicians' use of breast cancer risk assessment tools according to their perceived importance of breast cancer risk factors: an international survey. J Community Genet. 2019 Jan;10(1):61-71.

21 Lee AJ, Cunningham AP, Kuchenbaecker KB, Mavaddat N, Easton DF, Antoniou AC; Consortium of Investigators of Modifiers of BRCA1/2; Breast Cancer Association Consortium. BOADICEA breast cancer risk prediction model: updates to cancer incidences, tumour pathology and web interface. $\mathrm{Br} \mathrm{J}$ Cancer. 2014 Jan;110(2):535-45.

22 Meindl A, Ditsch N, Kast K, Rhiem K, Schmutzler RK. Hereditary breast and ovarian cancer: new genes, new treatments, new concepts. Dtsch Arztebl Int. 2011 May; 108(19):323-30.

23 Katz SJ, Ward KC, Hamilton AS, Mcleod MC, Wallner LP, Morrow M, et al. Gaps in Receipt of Clinically Indicated Genetic Counseling After Diagnosis of Breast Cancer. J Clin Oncol. 2018 Apr;36(12):1218-24.

24 Delikurt T, Williamson GR, Anastasiadou V, Skirton H. A systematic review of factors that act as barriers to patient referral to genetic services. Eur J Hum Genet. 2015 Jun;23(6): 739-45.

25 Owens DK, Davidson KW, Krist AH, Barry MJ, Cabana M, Caughey AB, et al.; US Preventive Services Task Force. Risk assessment, genetic counseling, and genetic testing for BRCA-related cancer: US Preventive Services Task Force recommendation statement. JAMA. 2019 Aug;322(7):652-65.

26 Katz SJ, Bondarenko I, Ward KC, Hamilton AS, Morrow M, Kurian AW, et al. Association of Attending Surgeon with Variation in the Receipt of Genetic Testing after Diagnosis of Breast Cancer. JAMA Surg. 2018 Oct;153(10): 909-16.
27 Heemskerk-Gerritsen BA, Rookus MA, Aalfs CM, Ausems MG, Collée JM, Jansen L, et al.; HEBON. Improved overall survival after contralateral risk-reducing mastectomy in BRCA $1 / 2$ mutation carriers with a history of unilateral breast cancer: a prospective analysis. Int J Cancer. 2015 Feb;136(3):668-77.

28 Van Allen EM. The Potential and Challenges of Expanded Germline Testing in Clinical Oncology. JAMA. 2017 Sep;318(9):801-3.

29 Kurian AW, Lichtensztajn DY, Keegan TH, Nelson DO, Clarke CA, Gomez SL. Use of and mortality after bilateral mastectomy compared with other surgical treatments for breast cancer in California, 1998-2011. JAMA. 2014 Sep;312(9):902-14.

30 Finch AP, Lubinski J, Møller P, Singer CF, Karlan B, Senter L, et al. Impact of oophorectomy on cancer incidence and mortality in women with a BRCA1 or BRCA2 mutation. J Clin Oncol. 2014 May;32(15):1547-53.

31 Bosse K, Rhiem K, Wappenschmidt B, Hellmich M, Madeja M, Ortmann M, et al. Screening for ovarian cancer by transvaginal ultrasound and serum CA125 measurement in women with a familial predisposition: a prospective cohort study. Gynecol Oncol. 2006 Dec;103(3):1077-82.

32 NCCN Guidelines Insights: Genetic/Familial High-Risk Assessment: Breast, Ovarian, and Pancreatic, Version 1.2020. J Natl Compr Canc Netw. 2020 Apr;18(4):380-91.

33 Robson ME, Bradbury AR, Arun B, Domchek SM, Ford JM, Hampel HL, et al. American Society of Clinical Oncology Policy Statement Update: Genetic and Genomic Testing for Cancer Susceptibility. J Clin Oncol. 2015 Nov; 33(31):3660-7. 